\title{
PROJECTIVE RESOLUTIONS FOR GRAPH PRODUCTS
}

\author{
by DANIEL E. COHEN
}

(Received 28th October 1993)

\begin{abstract}
Let $\Gamma$ be a finite graph together with a group $G_{v}$ at each vertex $v$. The graph product $G(\Gamma)$ is obtained from the free product of all $G_{v}$ by factoring out by the normal subgroup generated by $\left\{g^{-1} h^{-1} g h ; g \in G_{v}, h \in G_{w}\right\}$ for all adjacent $v, w$.

In this note we construct a projective resolution for $G(\Gamma)$ given projective resolutions for each $G_{v}$, and obtain some applications.
\end{abstract}

1991 Mathematics Subject Classification: $20 \mathrm{~J} 05$.

Let $\Gamma$ be a finite graph together with a group $G_{v}$ at each vertex $v$. The graph product $G(\Gamma)$ is obtained from the free product of all $G_{v}$ by factoring out by the normal subgroup generated by $\left\{g^{-1} h^{-1} g h ; g \in G_{v}, h \in G_{w}\right\}$ for all adjacent $v, w$.

In this note we construct a projective resolution for $G(\Gamma)$ given projective resolutions for each $G_{v}$, and obtain some applications. The applications are already known, but the current setting provides extra insight into the situations discussed. The construction is quite easy, since $G(\Gamma)$ is built up from the vertex groups by direct products and amalgamated free products. For ease of notation, we denote $G(\Gamma)$ simply by $G$.

To each vertex group $G_{v}$ we take a projective resolution by right modules for which the zero-dimensional module is the group ring. We denote this resolution by $\mathbf{Q}_{v} \rightarrow R G_{v} \rightarrow$ $R \rightarrow 0$.

We will need to choose an ordering of the vertices. Let $K$ be a complete subgraph of $\Gamma$, whose vertices, in the chosen order, are $v_{1}, \ldots, v_{k}$. Then $G(K)=G_{v_{1}} \times \cdots \times G_{v_{k}}$. The tensor product $\mathbf{Q}_{v_{1}} \otimes_{R} \cdots \otimes_{R} \mathbf{Q}_{v_{k}}$ is an $R G(K)$-module, and we define $\mathbf{P}_{K}$ to be $\left(\mathbf{Q}_{v_{1}} \otimes_{R} \cdots \otimes_{R} \mathbf{Q}_{v_{k}}\right) \otimes_{R G(K)} R G$. This, of course, stands for a sequence of $R G$-modules.

Let $C(\Gamma)$ be the set of all complete subgraphs of $\Gamma$. Define $P(\Gamma)$ to be the direct sum $\sum_{K \in C(\Gamma)} \mathbf{P}_{K}$. The boundary operators in the projective resolutions of the vertex groups give, in the usual way, boundary operators in tensor products. This gives rise to a boundary operator in $\mathbf{P}(\Gamma)$. Because our notation is such that the boundary of a onedimensional element of $\mathbf{Q}_{\nu}$ is not in $\mathbf{Q}_{v}$, we see that $\mathbf{P}_{K}$ is not a subcomplex of $\mathbf{P}(\Gamma)$; however, $\sum_{L \subseteq K} \mathbf{P}_{L}$ is a subcomplex.

Theorem. $\mathbf{P}(\Gamma) \rightarrow R G \rightarrow R \rightarrow 0$ is a projective $R G$-resolution for the trivial module $R$. 
We will give some applications of the theorem before proving it. The following corollary is immediate.

Corollary 1. Let the vertex groups of a graph product over a finite graph all be $F P_{n}$ ( for any $n \leqq \infty$ ), of finite cohomological dimension, or FP or FL. Then the graph product has the same property.

Chiswell [4] defines an Euler characteristic for any FP group, and he obtains in [5] a formula for the Euler characteristic of a graph product, using the inductive decomposition of a graph product in terms of amalgamated free products and direct products. Our theorem (which relies on this decomposition) makes it clearer where Chiswell's formula comes from. However, his proof also holds for Brown's definition [3] of the Euler characteristic, which ours does not appear to.

Let $G$ be a group, and let $P$ be a finitely generated projective $R G$-module. Then Hattori [7] and Stallings [8] define a trace function $t_{P}$, which is an element of $R\left(G / G^{\prime}\right)$. Let $\tau_{P}$ be the coefficient of 1 in $t_{P}$. When $G$ is $F P$ take a projective resolution $P$ which is finitely generated in all dimensions and zero in all but finitely many. Chiswell defines the Euler characteristic of $G$ to be $\sum_{0}^{\infty}(-1)^{i} \tau_{P_{i}}$.

Let $G$ be a subgroup of $H$, and let $P$ be a finitely generated projective $R G$-module. Then $P \otimes_{R G} R H$ is a finitely generated projective $R H$-module, and $t_{P \otimes_{R G} R H}$ is the image in $R\left(H / H^{\prime}\right)$ of $t_{P}$; hence $\tau_{P \otimes_{R G} R H}=\tau_{P}$. Also, if $Q$ is a finitely generated projective $R F$-module, for some group $F$, then $P \otimes_{R} Q$ is a finitely generated projective $(G \times F)$ module, and $t_{P \otimes_{R} Q}=t_{P} \otimes t_{Q}$ in $R(G \times F) /(G \times F)^{\prime}=R\left(G / G^{\prime}\right) \otimes \otimes_{R} R\left(F / F^{\prime}\right)$; hence $\tau_{P \otimes_{R} Q}=$ $\tau_{P} \tau_{Q}$. These facts can be found in [2], and are easy to prove directly.

Let $\Gamma$ be a finite graph, with an $F P$ group at each vertex. For each complete subgraph $K$ of $\Gamma$, with vertices $v_{1}, \ldots, v_{k}$, let $\chi_{K}=\left(\chi_{v_{1}}-1\right) \ldots\left(\chi_{v_{k}}-1\right)$, where $\chi_{v_{i}}$ is the Euler characteristic of the group at the vertex $v_{i}$. From our main theorem and the remarks in the previous paragraph, we immediately deduce the following formula due to Chiswell [5] (our formula differs slightly from his, because he includes the empty subgraph and we do not).

Corollary 2. With the above notation, $\chi(G T)-1=\sum \chi_{k}$, the sum being taken over all complete subgraphs of $\Gamma$.

Let $\langle Y ; S\rangle$ be a presentation of a group. This group then has a free resolution whose basis in dimension 1 is (a set bijective with) $Y$, and whose basis in dimension 2 is (a set bijective with) $S$. The boundary operator in dimension 2 can be described by means of the Fox derivatives.

Take such a presentation $\left\langle Y_{v} ; S_{v}\right\rangle$ for each vertex group of a graph product, and take the corresponding free resolutions. Then the graph product has a presentation $\left\langle\bigcup Y_{v}, \bigcup S_{v} \cup C\right\rangle$, where $C$ is the set of all $[a, b]$ for $a \in Y_{v}, b \in Y_{w}$ and all adjacent vertices $v$ and $w$ such that $v<w$ in the chosen ordering of the graph.

Consider the resolution of the graph product obtained by our theorem from the free resolutions of the vertex groups. It will be a free resolution whose basis in dimension 1 will be $\bigcup Y_{v}$. In dimension 2 it will have two kinds of basis elements. The members of 
$\bigcup S_{v}$ form the first kind, while the second kind consists of all $a \otimes b$, where $a \in Y_{v}, b \in Y_{w}$, for all adjacent vertices $v$ and $w$ such that $v<w$. Thus this basis is bijective with the basis given by the presentation of the graph product. Further, the boundary operator given by the theorem is exactly that given by the presentation.

In dimension 3 there are four kinds of basis elements. The first kind consists of all basis elements in dimension 3 of the resolutions of the vertex groups. The second kind consists of all $a \otimes s$ where $a \in Y_{v}, s \in S_{w}$, for all adjacent vertices $v$ and $w$ such that $v<w$. The third kind consists of all $s \otimes b$ where $s \in S_{v}, b \in Y_{w}$, for all adjacent vertices $v$ and $w$ such that $v<w$. The fourth kind consists of all $a \otimes b \otimes c$ where $a \in Y_{u}, b \in Y_{v}, c \in Y_{w}$ and $\{u, v, w\}$ is a complete subgraph with $u<v<w$.

The boundary operator can be explicitly described for the basis elements of the second, third, and fourth kinds. When this is done, we recover a result of Baik, Howie and Pride [1].

Proof of Theorem. It is easy to see, by standard results, that the relevant modules are projective, and we need only show that the sequence is exact.

We begin with the case when $\Gamma$ is complete, and first assume that it has only two vertices, $u$ and $v$. It is well-known (see, for instance, [3, V.1.1]) that there is a projective resolution

$$
\left(\mathbf{Q}_{u} \otimes_{R} \mathbf{Q}_{v}\right) \oplus\left(\mathbf{Q}_{u} \otimes_{R} R G_{v}\right) \oplus\left(R G_{u} \otimes_{R} \mathbf{Q}_{v}\right) \rightarrow R G \rightarrow R \rightarrow 0 .
$$

Then our result follows from the isomorphisms

$$
\begin{gathered}
\mathbf{Q}_{u} \otimes_{R} \mathbf{Q}_{v} \cong\left(\mathbf{Q}_{u} \otimes_{R} \mathbf{Q}_{v}\right) \otimes_{R G} R G \\
\mathbf{Q}_{u} \otimes_{R} R G_{v} \cong \mathbf{Q}_{u} \otimes_{R G_{u}} R G \\
R G_{u} \otimes_{R} \mathbf{Q}_{v} \cong \mathbf{Q}_{v} \otimes_{R G_{v}} R G .
\end{gathered}
$$

When $\Gamma$ is complete on the vertex set $v_{1}, \ldots, v_{k}$, with $k>2$, the result is immediate by induction, as $G=\left(G_{v_{1}} \times \cdots \times G_{v_{k-1}}\right) \times G_{v_{k^{\prime}}}$.

Now suppose that $\Gamma$ is not complete, and that its vertex set is $V$. Then there will be a pair of vertices $x, y$ which are not adjacent. Let $\Gamma_{0}, \Gamma_{1}, \Gamma_{2}$ be the full subgraphs whose vertex sets are $V-\{x, y\}, V-\{y\}, V-\{x\}$ respectively; let $G_{0}, G_{1}, G_{2}$ be the corresponding graph products. It is easy to see that $G=G_{1}{ }^{*} G_{0} G_{2}$.

We can assume, inductively, that the theorem holds for $\Gamma_{i}(i=0,1,2)$. Since the functor $-\otimes_{R G_{i}} R G$ is exact, we have long exact sequences

$$
\sum_{K \in C\left(\Gamma_{i}\right)} \mathbf{P}_{K} \rightarrow R G_{i} \otimes_{R G_{i}} R G \rightarrow R \bigotimes_{R G_{i}} R G \rightarrow 0 .
$$

We then have a commutative diagram 


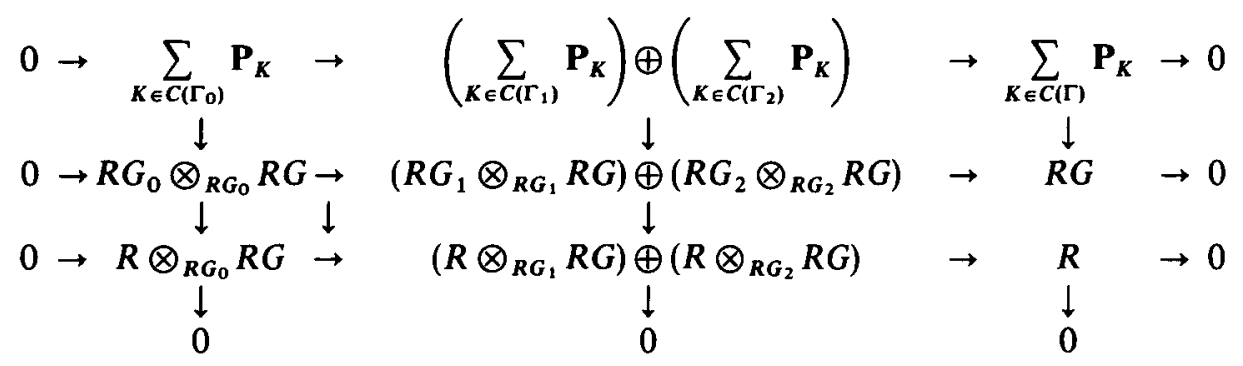

Here, the top row stands for a sequence of modules and maps. The left and middle colums are exact (when expanded out into such a sequence), by the inductive assumption. The middle row is trivially exact. The top row is also exact (when expanded out, this means that it is exact in each dimension), since $C\left(\Gamma_{0}\right)=C\left(\Gamma_{1}\right) \cap C\left(\Gamma_{2}\right)$, obviously, while $C(\Gamma)=C\left(\Gamma_{1}\right) \cup C\left(\Gamma_{2}\right)$, since any complete subgraph must lie in $\Gamma_{1}$ or in $\Gamma_{2}$, because $x$ and $y$ are not adjacent.

The bottom row was shown to be exact by Swan [9]. By standard homological algebra, the right column is also exact, as required.

Acknowledgement. I thank the referee for suggesting a clearer proof of the theorem than my original proof.

\section{REFERENCES}

1. Y.-G. Baik, J. Howie and S. J. Pride, The identity problem for graph products, J. Algebra 162 (1993), 168-177.

2. H. Bass, Euler characteristics and characters of discrete groups, Invent. Math. 35 (1976), 155-196.

3. K. S. Brown, Cohomology of groups (Graduate texts in mathematics 87, Springer-Verlag, 1982).

4. I. M. Chiswell, Euler characteristics of groups, Math. Z. 147 (1976), 1-11.

5. I. M. CHIswell, The Euler characteristic of graph products and Coxeter groups, Discrete groups and geometry (W. J. Harvey and C. MacLachlan, eds., London Mathematical Society Lecture Notes 173, Cambridge University Press, 1992), 36-46.

6. E. R. Green, Graph products of groups (Ph.D. Thesis, University of Leeds, 1990).

7. A. Hattorı, Rank elements of a projective module, Nagoya Math. J. 25 (1965), 113-120.

8. J. R. Stallings, Centerless groups-an algebrain formulation of Gottlieb's theorem, Topology 4 (1965), 129-134.

9. R. G. Swan, Groups of cohomological dimension one, J. Alg. 12 (1969), 585-610.

School of Mathematical Sciences

Queen Mary and Westfield College

Mile END RoAd

LONDON EI 4NS

ENGLAND

E-mail address: D.E.Cohen@qmw.ac.uk 